\title{
Theorietage der Gesellschaft für Informatik in Speyer 2015-Special Issue
}

\author{
Henning Fernau
}

FB 4, Informatikwissenschaften, University of Trier, Universitätsring, 54296 Trier, Germany; fernau@uni-trier.de; Tel.: +49-651-201-2827; Fax: +49-651-201-3958

Academic Editor: Tatsuya Akutsu

Received: 27 August 2016; Accepted: 20 September 2016; Published: 26 September 2016

Abstract: We briefly report on the national workshops on Formal Languages and Automata Theory as well as on Algorithms and Complexity Theory held in early Autumn, 2015.

Keywords: formal languages; parsing; tree automata; NP-hardness; parameterized complexity

"Gesellschaft für Informatik" (or GI for short) is the name of the German Computer Society. As in the case of its "big sister" ACM, it is divided into several sections and branches. For instance, the section on Foundations of Computer Science is split into several Special Interest Groups (SIGs), among them, one on Formal Languages and Automata Theory and one on Algorithms and Complexity Theory. Both SIGs have their own regular meetings, either annually or even more frequently. In 2015, these meetings were held together for the first time, organized by the group of Theoretical Computer Science from the University of Trier. This joint meeting was held in Speyer, one of the most important medieval German cities on the river Rhine.

This type of common meeting is not that usual, given the fact that, traditionally, Algorithms and Complexity Theory is classified as "track A" in conferences like ICALP, while Formal Languages and Automata Theory is part of "track B". However, there is some overlap between these areas, which might be rather hidden these days, also due to the aforementioned classification, or better said segmentation, of (Theoretical) Computer Science.

To better bridge both areas, we invited five distinguished speakers to give talks of one hour each on one day, situated between the meetings of both aforementioned SIGs. We gratefully acknowledge the financial support by GI and MDPI that allowed us to have these five talks.

- Till Tantau, Lübeck, Germany: Algorithmic metatheorems 2.0;

- Ralf Treinen, Paris, France: Towards reasoning about file trees using tree automata;

- Christian Komusiewicz, Berlin, Germany: Multivariate algorithmics for NP-hard string problems;

- Henrik Björklund, Umeå, Sweden: On the complexity of mildly context-sensitive formalisms;

- Laura Kallmeyer, Düsseldorf, Germany: LCFRS: Parsing and applications in computer linguistics.

As all these talks were very well received, we decided to invite all of the speakers to submit extended versions of their papers to this Special Issue, so that the material can be accessed by a wider international readership. Four of the speakers agreed to this invitation, and hence this Special Issue collects the following contributions, sorted by the number assigned to the article in this journal.

21 Christian Komusiewicz: Multivariate Algorithmics for Finding Cohesive Subnetworks [1]

32 Henrik Björklund, Martin Berglund and Petter Ericson: Uniform vs. Nonuniform Membership for Mildly Context-Sensitive Languages: A Brief Survey [2]

44 Till Tantau: A Gentle Introduction to Applications of Algorithmic Metatheorems for Space and Circuit Classes [3]

58 Laura Kallmeyer, Wolfgang Maier: LR Parsing for LCFRS [4] 
The first listed paper differs from the one that Christian Komusiewicz presented in Speyer, because that talk was based on a survey that he had recently published in the EATCS Bulletin in 2014, see [5].

We believe that all contributions show that the basic areas of Theoretical Computer Science still have very strong links to each other, and we hope that the readers of this Special Issue will understand and enjoy this very particular flavor. All papers have a survey-type character that should make them accessible to a wider readership. We also hope that these articles inspire the readers to attend workshops or conferences with special emphasis on well chosen (survey) talks.

Finally, we would like to direct the reader to the complete program of the workshops (including short summaries of the talks) that is available as a Technical Report of the University of Trier [6].

Acknowledgments: We thank all contributors to this Special Issue, the referees for their scholarly and timely work and all members of the Steering Committee of the GI-SIG "Automata and Formal Languages" for their support.

Conflicts of Interest: The author declares no conflict of interest.

\section{References}

1. Komusiewicz, C. Multivariate Algorithmics for Finding Cohesive Subnetworks. Algorithms 2016, 9, 21.

2. Björklund, H.; Berglund, M.; Ericson, P. Uniform vs. Nonuniform Membership for Mildly Context-Sensitive Languages: A Brief Survey. Algorithms 2016, 9, 32.

3. Tantau, T. A Gentle Introduction to Applications of Algorithmic Metatheorems for Space and Circuit Classes. Algorithms 2016, 9, 44.

4. Kallmeyer, L.; Maier, W. LR Parsing for LCFRS. Algorithms 2016, 9, 58.

5. Bulteau, L.; Hüffner, F.; Komusiewicz, C.; Niedermeier, R. Multivariate Algorithmics for NP-Hard String Problems. Bull. EATCS 2014, 114.

6. Fernau, H. Theorietage 2015 Algorithmen und Komplexität \& Automaten und Formale Sprachen (Speyer, 29 September-2 October 2015); Technical Report; Mathematics/Computer Science: Trier, Germany, 2015.

(C) 2016 by the author; licensee MDPI, Basel, Switzerland. This article is an open access article distributed under the terms and conditions of the Creative Commons Attribution (CC-BY) license (http://creativecommons.org/licenses/by/4.0/). 